\title{
Place Brand Satisfaction Elements
}

The formation of image has been described by Reynolds (1965, p. 69), as one of the first commentators, as the development of a mental construct based on a few impressions chosen from a flood of information. In the case of place image, this 'flood of information' has many sources, including promotional sources (advertising and brochures), the opinions of others (family/friends, travel agents), media reporting (newspapers, magazines, television news reporting and documentaries) and popular culture (motion pictures, literature); 'Furthermore, by actually visiting the destination, the image will be affected and modified based upon first hand information and experience' (Echtner and Ritchie 2003, p. 38).

As Reynolds (1965, p. 70) states: "often, of course, the word "image" is used as equivalent to reputation ... what people believe about a person or an institution, versus character, what the person or institution actually is'. The latter could also be referred to as identity, as discussed in Chapter 4. We shall focus now on the image as the mental construct of the tourist, migrant worker, investor or trader. The following sections will expand on what Ryan (2000, p. 121) states the visitor to be; that is, 'a voyeur whose very presence is a catalyst for action in both the meta and narrow narrative; an interpreter of experience within personal constructs of meaning, but able to discard those meanings in ludic moments ... Within this framework of analysis the place becomes a locus of selected meanings'. However, a challenge for hosts that attract culturally different groups of visitors is to exploit those few impressions in order to mould perceptions and thereby raise the right expectations. The further away and the more culturally different the visitor, the harder the task of influencing the perceived place image will be. As explained by McCabe and Stokoe (2004, p. 604): 'The changing nature and character of places, together with adapting modes of leisure consumption and commercialization of locales, have shifted the ways in which 
meanings are attached to places.' The way in which people do this is the subject of the following section.

\section{PERCEIVED PLACE IMAGE}

With regard to experiential products, people are involved in an ongoing search for information (Leemans 1994, p. 23). By collecting information, the consumer creates an image or 'mental prototype'(Tapachai and Waryszak 2000, p. 37) of what the experience might be like. As place experiences are intangible, images become more important than reality (Gallarza et al. 2002, p. 57) and the place images projected in information space will have a great influence on the place images as perceived by consumers. The latter are generally accepted (Echtner and Ritchie 1991, 1993, 2003; Padgett and Allen 1997, p. 50; Tapachai and Waryszak 2000, p. 38) to be based on attributes, functional consequences (or expected benefits), and the symbolic meanings or psychological characteristics consumers associate with a specific place (or service). As a consequence, projected images influence place positioning and ultimately location choice behaviour.

There appears to be a consensus among authors that the place image research stream has emerged from Hunt's work of 1971 (Gallarza et al. 2002, p. 58; Hunt 1975). 'From this time onwards, there have been numerous and varied approaches to its study', totalling 65 works, between 1971 and 1999, as identified by the thorough synoptic work of Gallarza et al. (2002) and of Pike (2002), who reviewed 142 papers. One influential study was published by Echtner and Ritchie (1991, 1993, 2003). Through their research, Echtner and Ritchie concluded that:

- Place image should be envisioned as having two main components: those that are attribute based and those that are holistic.

- Each of these components contains functional (or more tangible) and psychological (or more abstract) characteristics.

- Place images can also range from those based on 'common' functional and psychological traits to those based on more distinctive or even unique features, events, feelings or auras.

This illustrates that there are many aspects involved in formulating the total place image in the mind of the (potential) visitor. The three-dimensional model envisaged by Echtner and Ritchie is depicted in Figure 11.1, together with some examples for four of the six components. The common-versusunique dimension is missing in this example for Nepal, but it normally 
FIGURE 11.1 Components of place image and an example of four of these for Nepal

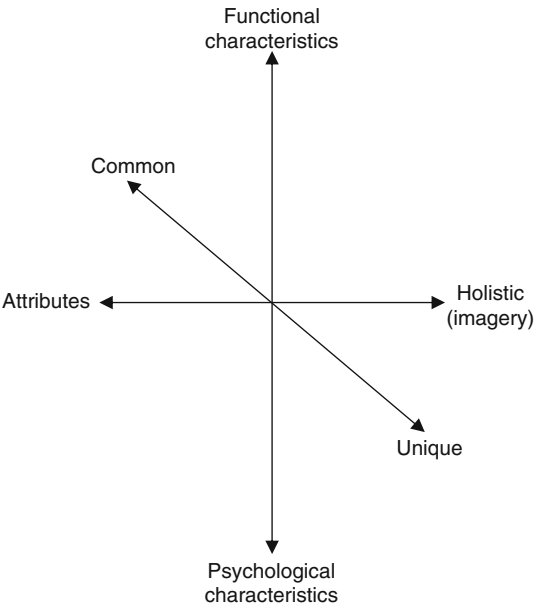

Components of place image

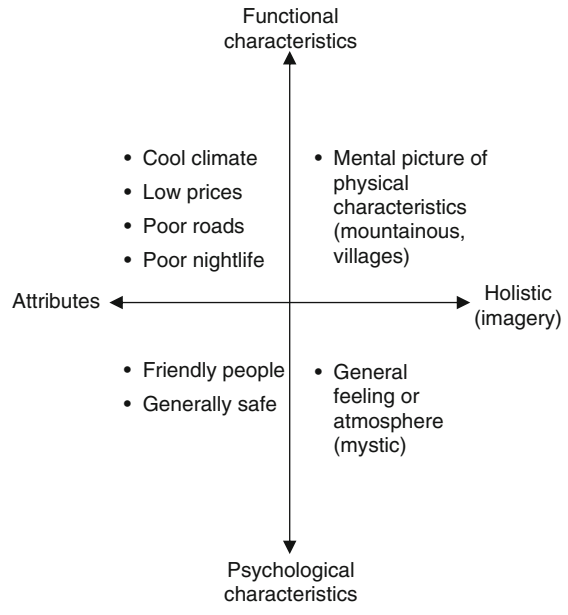

Place image of Nepal

Source: Reproduced from Echtner and Ritchie (1991), with the kind permission of the Journal of Tourism Studies.

identifies whether image aspects are unique for the specific destination, or shared by others as well.

It is apparent that, similar to the earlier discussion about the 'true' identity of place (see the section on Identity and Culture in Chapter 4), 'the' place image does not really exist either. Different projections and perceptions are individual or community constructions, and different individuals and communities might have different or fragmented insights. Arguably, it would be better to refer to the 'dominant view', which would normally correspond more or less to the identity of place in line with sociologist Stuart Hall's narrative of the nation. Therefore, when we refer to 'the' place image, what is really meant is the 'dominating image', or the tendency to stereotype place. Nevertheless, for the sake of readability, we refer mainly to 'the' image, as if it were a single concept, keeping in mind that it is in fact an individualized construct, which incorporates many variations and interpretations.

Because of the complexity of the construct of place image, Echtner and Ritchie (1991, 1993, 2003) proposed a combination of structured and unstructured methodologies to measure place image. They proposed open-ended questionnaires to capture holistic components and more distinctive or unique features of the place image. Second, an attribute-based 


\section{TABLE 11.1 A taxonomy of procedures for measuring place image}

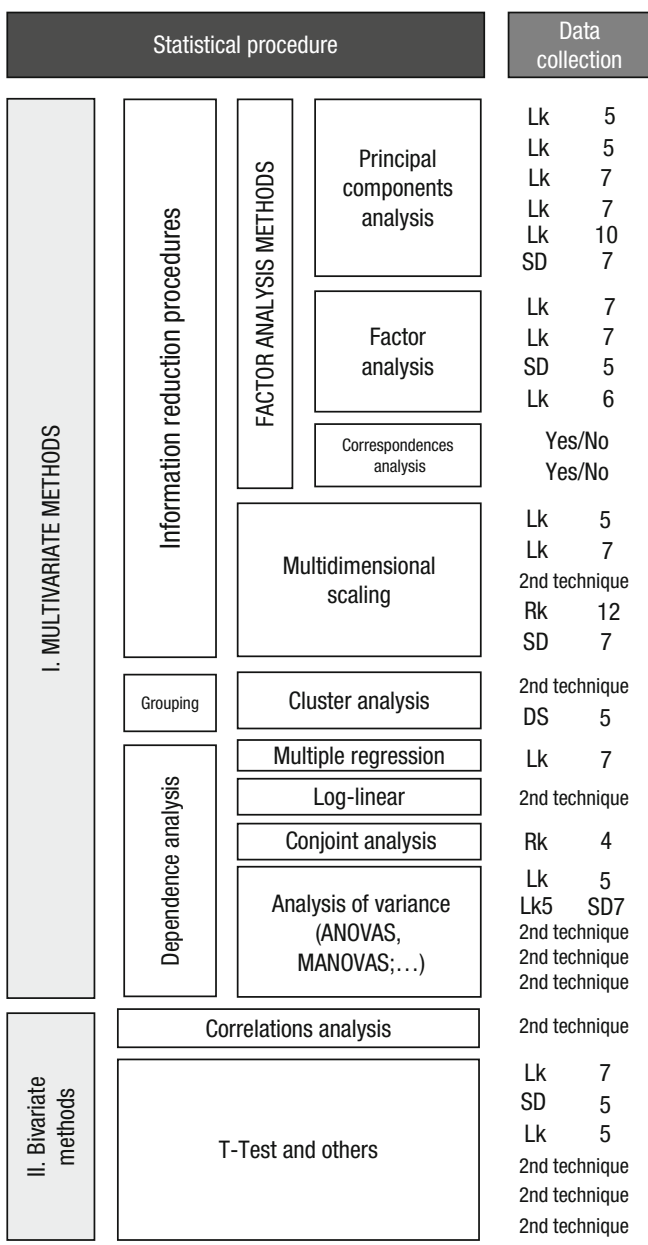

Authors

\author{
Ahmed $(1991 ; 1996)$ Baloglu (1997) \\ Baloglu \& McCleary (1999) \\ Walmsley \& Young (1998) Sternquist (1985) \\ Fakeye \& Crompton (1991) \\ Muller (1995) \\ Driscoll et al. (1994)
}

Crompton et al. (1992) Schroeder (1996) Opperman (1996a, 1996b) Guthrie \& Gale (1991)

Crompton (1979)

Echtner \& Ritchie (1993)

Calantone et al. (1989)

Eizaguirre \& Laka (1996)

Gartner (1989)

Goodrich (1982)

Guthrie \& Gale (1991)

Haahti (1986)

Baloglu \& Brinberg (1997)

Muller (1995)

Embacher \& Buttle (1989)

Dadgostare Isotalo (1995)

Eizaguirre \& Laka (1996)

Carmichael (1992)

Chon (1992)

Baloglu \& McCleary (1999)

Schroeder (1996) Crompton (1979)

Gartner \& Hunt (1987) Baloglu (1997)

Fakeye \& Crompton (1991 Ahmed (1991; 1996)

Dadgostare Isotalo (1995)

Chon (1991)

Gartner \& Hunt (1987)

Borchgrevink \& Knutson (1997)

Fakeye \& Crompton (1991) Driscoll et al. (1994) Ahmed (1991) Muiller (1995) Reilly (1990)

Opperman (1996a, 1996b) Schroeder (1996)

Notes: SD = semantic differential; Lk = Likert scale; Categorical data: Yes/No; Rk = Ranking order. When studies developed successive algorithms of the transformed data, the name of the author(s) appears repeated in two or more sections. In these cases, the data collection method is cited with the first technique and '2nd technique' appears in the data collection column, in the second citation.

Source: Table and listed references reprinted from Gallarza et al. (2002), with the kind permission of Elsevier. (Full details of the references can be found there.)

eight-factor scale was produced to measure place image performance across destinations. Most studies to date emphasize only the second attribute-based approach to assessing place image, as is illustrated in Table 11.1. Gallarza et al. $(2002$, p. 67) conclude that: 'for the most part, there is 
a combination of multivariate and bivariate techniques, with a greater or lesser presence of qualitative techniques in the preliminary steps. Very few studies use qualitative methods as the main technique. Among all collection procedures, the seven-point Likert Scale is the most commonly used'. Such studies must, however, be limited because they cannot capture the holistic nature and subjective perspective of the individual, nor a place's unique characteristics of the image (Echtner and Ritchie 2003; Tapachai and Waryszak 2000). As Bigné et al. (2001, p. 611) state: 'the sum of the attribute scores is not an adequate measurement of the overall image'. The ideas presented by Van Riel (1996) in the corporate image domain, referred to in Chapter 2, would support this reasoning, as one would at least need to take into account the level of consumer elaboration of the image.

While Echtner and Ritchie (1993, p. 12) claim that their approach can be used to compare and contrast most, if not all, place images, one needs to realize that places and their types of offering can be classified into many different categories: as a mass versus niche tourism destination; as a meeting, conference or exhibition location; a cultural hotspot; or as a business, investment or employment hub. When considering all common and unique image characteristics, each category has a long list of specific attributes attached to it. Morgan (1999), for example, created a rating system for beach destinations that included forty-nine attributes for beaches alone. The categorization of destinations is normally set by the researcher (with very few studies comparing two or more different types of places), but consumers' perceptions of what type of place is being considered and what the boundaries are, might not be so clear (Gallarza et al. 2002, p. 65). Cho and Fesenmaier (2000) targeted their enquiry on small-scale eco-tourism as the object of their virtual tour research. The ingredients for such a virtual eco-tour would be relatively easy to assess. However, would it also be applicable in a metropolitan context - if, for example, the Amsterdam or London place marketers decided to create a virtual tour to promote their 'product offering'? Within the metropolitan context, the list of potential attributes to be considered would be much more complex. And to add to the confusion, what if a place such as Dubai were to be included in such a study? It has developed an impressive metropolitan presence, but attracts many tourists as a beach resort, though it offers cultural attractions and opportunities to escape into the desert, as well tremendous investment, employment and trade opportunities. How would one assess the image to be projected in a virtual tour for a place like that?

In recent years, calls for more pluralistic approaches to understanding place image formation have become louder (Feighey 2003; Jenkins 1999; MacKay and Couldwell 2004). Some, for example, have begun to use photographs (Dann 1996b; MacKay and Couldwell 2004; MacKay and Fesenmaier 1997; Markwell 1997). In the research on retail store image, the use of scales has 
long been criticized, based on the observation that 'people are encouraged to respond to characteristics that do not necessarily comprise the image they have of the store being studied' (Kunkel and Berry 1968, p. 25), and 'It has been suggested that a more appropriate measurement would be achieved by the use of unstructured instruments, followed by content analysis and coding of responses' (McDougall and Fry 1974, p. 54). In this way, 'the respondent is free to discuss only that which is relevant to his image of the store' (Kunkel and Berry 1968, p. 25). Related and alternative methods have been deployed in the research projects as presented in several of the case study chapters in this book, as we believe, as stated earlier, that holistic experiential and unique aspects of image are much more important than common attributes.

\section{VISITOR IDENTITY AS IMAGE SELF-FOCUS}

The interpretation of the many aspects involved in the formulation of place image in the mind of the visitor will differ between places and the types of visitors they attract (attributes differ according to object (type of place) and subject (person), as Gallarza et al. (2002, p. 62) put it). The latter is also referred to in the literature as 'self-focus', where the affective evaluations are not a description of the object, but of the relationship between the person and the object (Leemans 1994). Sirgy and Su (2000) emphasize the importance of 'self-congruity' it this context, which involves a process of matching a visitor's self-concept to the place image. A study by MacKay and Fesenmaier (2000) provides empirical evidence to support the notion that the manner in which people view place images is mediated by cultural background, for one thing. Cultural background determines people's values, ideals, norms, beliefs and folk wisdom (Arnould et al. 2003, p. 77). It would seem logical to assume that these will in turn have an impact on people's perceptions of places. The classic work by Hofstede (2001b), for example, found important differences in work-related values across forty national cultures.

It is generally accepted in the literature that, in the people's minds, the perceived place image is formed by two (Baloglu and Brinberg 1997; Baloglu and McCleary 1999; Embacher and Buttle 1989) or three (Dann 1996b; Gartner 1993) 'distinctly different but hierarchically interrelated components: cognitive, affective and [according to some] conative' (Gartner 1993, p. 193). These are all processes of awareness, according to Csikszentmihalyi (1995a, p. 19), who also identified attention (senses) and memory as the other two structural elements of human consciousness; 'Cognitive evaluations refer to the beliefs or knowledge about place attributes whereas affective evaluation refers to feelings toward, or attachment to it' (Baloglu and McCleary 1999, p. 870). According to Gartner (1993, p. 196), 'the affective 
component of image is related to the motives one has for destination selection'. The conative component, on the other hand, 'is the action component which builds on the cognitive and affective stages' (Dann 1996b, p. 49).

If self-congruity occurs, perceived place image would surely also be mediated by socio-demographic characteristics, cultural background, personal identity and psychological consumer characteristics, which is supported by Baloglu and McCleary (1999, p. 870) (see also Consumer Behaviour (Arnould et al. 2003; Blackwell et al. 2000)). Beerli and Martín (2004a, p. 678; 2004b, p. 623) provide evidence for this, as they have established empirically that: (i) motivations (as affective psychological characteristics) influence the affective component of image; (ii) the experience of travel (as in learning as a psychological characteristic) has a significant relationship with cognitive and affective images; and (iii) the socio-demographic and personal characteristics (gender, age, level of education, occupation, country of origin and social class) influence the cognitive as well as the affective assessment of image. However, it has to be stated that these conclusions need to be treated with caution, as most hypotheses in Beerli and Martín's study were only partially maintained; a specific model of causality was not constructed, and generalization of results was only permitted within the context of the case study of Lanzarote.

Nevertheless, Echtner and Ritchie (1993, p. 9) do not disagree with the idea that measuring the common attributes of place image is not sufficient, as individuals can hold different interpretations of perceived image. They found that answers to open-ended questions were a rich additional source needed to complement scale items, as they provided more descriptive, distinctive and detailed impressions. For example, "when one scale item measures the degree of perceived friendliness, the open-ended questions revealed the differences in the way this friendliness was manifest - in Jamaica as outgoing and fun, whereas in Japan as reserved and formal' (Echtner and Ritchie 1993, p. 9).

Fairweather and Swaffield (2002, p. 294), also support the notion that there is significant variation in the way individuals interpret a uniform place image. Help in understanding this can be sought in the literature on destination choice processes in tourism studies. Major contributions to this have been made by Crompton (Ankomah et al. 1996; Um and Crompton 1990); and Woodside (Sirakaya and Woodside 2005; Woodside and Lysonski 1989). For a detailed overview of existing literature on destination choice modelling, see Sirakaya and Woodside (2005). The most commonly used model (one of 'the grand models' according to Sirakaya and Woodside (2005, p. 818)) involves five stages: (i) need recognition; (ii) information search; (iii) alternative evaluation and selection; (iv) purchase; and (v) outcomes (or post-purchase behaviour) 
(Blackwell et al. 2000, ch. 3). Dellaert (1999) distinguishes six phases: (i) research, information search; (ii) composition, or the mental integration of different travel components; (iii) transaction, or purchase; (iv) creation, or production; (v) consumption; and (vi) evaluation, or post-purchase behaviour. It could be assumed that perceived place image will change as people go through these various processes. At the same time, however, Decrop and Snelders (2004) found that travel planning is an ongoing process, entailing much adaptability and opportunism, where fantasy and emotions also play an important role. Hence this departs from traditional rational decision-making models and supports the notion that, with regard to experiential products, people are involved in an ongoing search for information (Leemans 1994, p. 23; Vogt and Fesenmaier 1998, p. 553). As a result, the need recognition, information search, and alternative evaluation and selection processes might prove to be more dynamic and non-sequential in the decision-making process of selecting the place to visit for leisure, business or employment, as they are in other nonhedonic product purchase decisions.

Another approach to place choice modelling involves choice sets (Um and Crompton 1990; Woodside and Lysonski 1989): 'The concept postulates that there is a funneling process which involves a relatively large initial set of destinations being reduced to a smaller late set, from which a final destination is selected' (Ankomah et al. 1996, p. 138). The choice set model is depicted in Figure 11.2. The late set (referred to by others as the 'evoked set' (Sirakaya and Woodside 2005, p. 825) includes those places a traveller considers as probable places to visit. This set can be subdivided into an action set, comprising those places on which a potential visitor has taken some action, such as requesting brochures or visiting websites; and an inaction set of places, for which the individual took no further action. If a person does not have sufficient information to make an evaluation on a certain place, it will ultimately be placed in the inert set. Places in individuals'

\section{FIGURE 11.2 1 Choice set model}

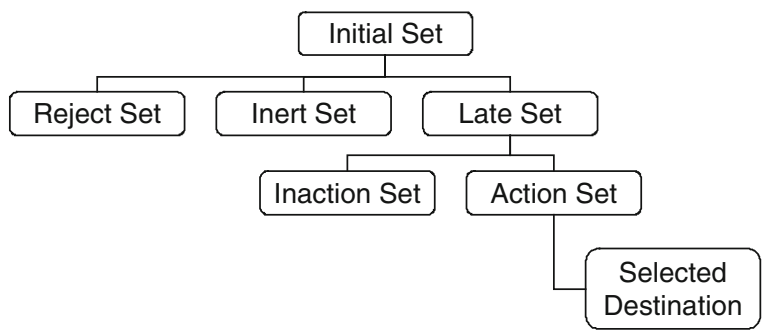


reject sets have been excluded from consideration because they have received a negative evaluation (Ankomah et al. 1996, p. 139).

It can be assumed that perceived place image changes as consumers move destinations from one set to another. This is also supported by Ankomah et al. (1996), who found empirical evidence to support the notion that the accuracy of cognitive distance estimation (as a possible component of perceived image - see Table 12.1) varies among choice sets. However, at the same time, Sirakaya and Woodside (2005, p. 829) propose that 'revisions occur to such consideration sets in a dynamic process as consumers move mentally toward making commitment and rejection decisions; [and] ... consumers are able to easily report intention probabilities to visit alternatives in consideration sets and these probabilities are revised dynamically'. Therefore, linking changes in perceived place image to altered choice sets will be a challenging task, and much of it is likely to depend on situational constraints (Sirakaya and Woodside 2005, p. 826). Not much research has been reported in this area, but our research on the case of the Netherlands presented in Chapter 12 will show that traditional attribute-based place image research seems to be incapable of establishing such a relationship between destination choice modelling and image. Therefore, evaluating alternative methodologies for measuring place image was a priority in the case research presented in this book.

As soon as effective standardized methodologies for measuring perceived place image become available (as illustrated above, much has been written but without achieving general agreement), evaluating the relationship between destination choice modelling and perceived place image will be a vast area of research. This, however, is not the focus of this book, and to avoid measurement issues relating perceived place image change to the progression within the different destination choice stages or formation of choice sets, the research presented in some of the case study chapters in this book will focus primarily on pre-choice perceived image in general (which in itself constitutes a gap in the existing literature). Because, as several authors have concluded, the evaluation of the place experience will certainly influence the image, and modify it (Bigné et al. 2001; Chon 1991; Echtner and Ritchie 1993; Fakeye and Crompton 1991).

\section{TEMPORAL ENVIRONMENTAL OR SITUATIONAL INFLUENCES}

Temporal environmental or situational influences will also change people's perceptions in the short term (Gartner and Hunt 1987) These are referred to as 'autonomous agents' by Gartner (1993, p. 201-3). Beerli and Martín (2004a, p. 667), based on the work of Gartner, attempted to measure the impact on place image of autonomous agents. They first highlighted guidebooks, news, 
articles, reports, documentaries and programmes about the place in the media. Later however, they combine these roughly into two variables related to 'tourist guidebooks' and 'news and popular culture'. In fact, news and popular culture were considered by Gartner (1993, p. 201) to be 'two [different] subcomponents in the autonomous category'. Obviously, these components can take many different forms and it would be impossible to try and list them exhaustively, as will be illustrated by the examples that follow.

The distinction between autonomous and induced agents might also not always be clear, as place marketers can influence the impact of temporal environmental and situational factors through public relations, crisis management and lobbying (Gartner 1993, p. 202). This was illustrated in Chapter 8, when we examined covert induced agents. The difference is that overt induced agents (as discussed in Chapter 4) are initiated by place marketers (or one or more of the place marketing stakeholders) as proactive interventions, while responses to autonomous agents can only be reactive. Finally, covert induced agents are somewhere in the middle, with place marketers attempting to influence otherwise autonomous processes that influence media agendas. This can be quite relevant, as Gartner (1993, p. 203) states: 'news and popular culture forms of autonomous image formation, because of their high credibility and market penetration, may be the only image formation agents capable of changing an area's image dramatically in a short period of time'. One of the reasons for this may be that news does not age well, and major events often receive massive attention in a relatively brief period of time. However, without reinforcement over time, images are likely to revert back to what they were before. This can be either positive or negative, but whatever the case, one would expect that place marketers would want to influence this process. That is why covert induced agents are becoming increasingly important, and the nature of many previously autonomous agents have been changed to become covert induced agents.

Nevertheless, Beerli and Martín (2004a) found that, as far as the autonomous sources are concerned, there seemed to be little impact on perceived image. However, again, it needs to be emphasized that Beerli and Martín measured only post-visit image. It is expected that autonomous agents will have a larger impact in pre-visit image formation, compared to post-visit perceptions, which are more likely to be influenced by organic agents (experience), as has been illustrated in earlier chapters. It will be obvious to most researchers that the role of the media, together with other temporal and environmental influences, cannot be ignored in the context of image formation. In particular with the internet and the current reach of mass media, and how people are influenced by these (Magala 2001), major events (significant political, economic, technological or social events) will 
affect what people observe and read. Subsequently, perceptions will change: 'One of the most common Autonomous image formation agents is news reporting. Generally destination area promoters have no control over what appears in a news story and the projected image is based on someone else's interpretation of what is happening in the area' (Gartner 1993, p. 201). Often, therefore, place marketing can be a demanding job.

Recent examples of the tragic impact that major news events and their coverage in the news can have on places are obvious. The 2004 Indian Ocean tsunami; the appalling terrorist attacks in various parts of the world; and the lethal SARS outbreak are, of course, examples that stand out. Sometimes it is not even the catastrophe itself that is to blame for deterring visitors, but the way it is blown out of proportion in the press. To illustrate this in case of SARS, tourism professionals were addressing the phenomenon with SIP (SARS Induced Panic) to illustrate that, not the virus itself, but the subsequent dramatization in the press was to blame for the rapid decline in international travel to the affected regions.

These situational and transient circumstances could be of great influence on place image, and therefore also on choice behaviour. However, many of these can be positive, unexpected and dormant. A good example was in the press in 2002. According to a survey of British tourist boards, the 'Queen Mother effect' had boosted the number of visitors to the UK and helped erase memories of the foot and mouth crisis. The Telegraph newspaper (e-tid 2002) reported that the results of the survey by MICG, a London-based tourism conference organizer, suggested that the Queen Mother's funeral had had more success than official marketing campaigns in encouraging visitors back to Britain. Bookings rose by nearly 20 per cent in Cumbria, one of the areas worst hit by foot and mouth decease, while Cornwall showed a similar increase, and many hotels reported occupancy levels close to pre-2001 rates. These examples illustrate how temporal, environmental or situational influences can have a dramatic short-term effect on perceived place image, and it is acknowledged that organizing mega-events such as the Olympic Games or World Cup soccer not only facilitate memorable place experiences, but they also have a dramatic effect on place image through the coverage they receive in the media (Nielsen 2001). Some of these changes and events will, of course, also have a longer-term impact on place image by changing a destination's identity, as described in the place brand strategy section in Chapter 4.

\section{SHARED MEANING THROUGH NARRATIVES}

Place image is formed in what Go and Van Fenema (2006) refer to as mind or knowledge space. 'We develop and express routines and experiences that are 
communicated ... through stories and scripts ... processed and enhanced by other human beings.' People interact and share meaning, and subsequently shape each others' perceptions of objects and places. In Gartner's terminology these are called Solicited or Unsolicited Organic Agents (1993, pp. 203-4) and referred to in Figure 3.1 as word of mouth and word of mouse (Riedl et al. 2002), the latter being a contemporary reference to people communicating and willingly or unwillingly collaborating online, such as through the use of email, newsgroups, collaborative filtering, personalized websites, blogs, chat rooms or audio/video and pictures (digitalized, accompanied or not by peoples comments, remarks and critiques); specifically, Web 2.0.

Because of the unique characteristics of services (such as their intangibility and the difficulty of quality control - see Chapter 4), consumer decisionmaking in tourism is often associated with perceived financial and emotional risks: 'In these high-risk situations, word-of-mouth or personal information sources are more influential than impersonal media sources' (Sirakaya and Woodside 2005, p. 827). In relation to these organic agents, Beerli and Martín (2004a, p. 677) state that 'the fact that word of mouth is considered to be the most believable and truthful communication channel, together with the fact that it also significantly influences the cognitive image, means that it is important that the messages transmitted in the markets of origin match the reality of the destination'. In other words, as we stated earlier, the projected image must be realistic. If not, public and private actors will struggle to satisfy visitors who arrive at their destination with glorified expectations (see also the Conclusion, below). This in turn will have a negative effect on the image they will transmit by word of mouth on their return home, causing clashes with other induced sources of image formation.

A lot of these processes now also occur online, for example, in virtual travel communities (Wang and Fesenmaier 2002, 2003, 2004a, 2004b; Wang et al. 2002). Dellaert (1999) argues that, with the internet, consumers become involved in the production and assemblage process, sharing information with others. The possibility of many-to-many communication could greatly affect branding strategies because it allows individuals to influence place images directly by sharing detailed experiential information with others. There should therefore be great potential in combining the enabling capabilities of expanded computer processing power and the internet with methodologies of content analysis as applied in the research area of narrative and social psychology. As places offer experiential products, as do most services (Dhar and Wertenbroch 2000; Padgett and Allen 1997; Vogt and Fesenmaier 1998), consumers try to organize a complex sequence of events and their reactions to these events (and the information gathered) into a meaningful whole. Understanding this is the province of narrative 
psychology, which contends that people have a natural propensity to organize information about experiences in story format. It also suggests that people relate their interpretations of experience to others by narrating, or telling stories. Nevertheless (as has been the case in place image research) the predominant explanation of psychological functioning has focused almost entirely on paradigmatic research, rather than considering the narrative mode. In other words, past research has considered consumers as rational thinkers rather than storytellers (Padgett and Allen 1997). Given the experiential nature of place visitation, this supports the notion that the assumptions of the narrative mode of thought may be more promising in place image research than those of the paradigmatic mode of thought, which is not divergent from current academic opinion (McCabe and Stokoe 2004; Padgett and Allen 1997; Tapachai and Waryszak 2000). The narratives people share will therefore be the focus of research when it comes to measuring the perceived place image of our signature case Dubai, as presented in Chapter 13.

\section{CONCLUSION: PLACE BRAND SATISFACTION GAP}

The result, at the end of this section, is a perceived place image built up from an extensive set of customer expectations, be it functional or psychological, attribute based or holistic, or based on common traits or unique features. The extent to which the actual place experience meets or exceeds these expectations will determine the level of visitor satisfaction (Bigné et al. 2001; Chon 1990; Govers and Go 1999). As MacInnes and Price (1987, p. 481) put it: 'even if the actual outcome is favourable, it is likely to differ from the imagined outcome. Deviations of the actual outcome from the imagined outcome give rise to surprise.' So one reason why visitors might leave the host dissatisfied may be because they are reading from a different script (Bateson 2002; Hubbert et al. 1995; Schoemaker 1996). In other words, their expectations might be unrealistic. These deviations are an important cause of customer dissatisfaction. So one way in which visitors become disappointed is when a place brand satisfaction gap occurs. This takes place when the perceived place image and the visitors' expectations are unrealistic and therefore clash with the real identity of the place and its product offering as experienced through consumption. This might occur if an unrealistic or incomplete place image has been projected, or people's interpretation of the place images is distorted because of temporal environmental or situational influences, interaction with others, or the person's own identity (selective attention and retention). This is illustrated by Fairweather and Swaffield (2002, p. 293), who found that place image 'also 
sets up criteria for negative evaluation. The promotional image is largely skewed towards a set of favourable experiences. When visitors encounter settings or experiences that differ markedly from their expectations, their evaluations can be very negative'. This is more likely to happen with culturally diverse groups of visitors, which would be supported by MacKay and Fesenmaier (2000). If the cultural setting of a place is completely different from that of the visitor's home country, the experience is more likely to hold surprises if the promotional image is skewed than in the case of a place where the culture is similar.

In another paper, Fesenmaier and MacKay (1996, p. 37) argue that a distorted image can also have a negative effect on the destination's residents: 'For residents, an image based on a false reality generates and perpetuates a lie with which the residents must live; thereby, robbing a culture of its authenticity.' This in itself will again have a negative effect on 'visitor satisfaction as place brand performance deteriorates. In other words, this illustrates that visitors might also become dissatisfied because the actual experience might not correspond to what was rightfully expected because of an inadequate place brand performance. Kitchin (2003, p. 17) calls this the 'belief gap'. This was discussed in more detail in Chapter 8 , but perhaps here we have to clarify this difference between the place brand satisfaction gap and the place brand performance gap.

Bigné et al. (2001) discuss in detail the relationship between perceived image and evaluative assessments such as perceived quality and customer satisfaction and their respective influence on post-purchase behaviour, measured by intention to return to and recommend a destination (much of this also based on the work by Oliver (1999) who discusses such issues within a wider marketing theory context). As Bigné et al. (2001, p. 608) argue, these aspects relate to the visitors' view rather than that of the providers. Hence it fits logically into the lower part of the place branding model, which was under scrutiny above (see Figure 3.1). The providers' perspective relates to the place brand strategy gap; the place brand satisfaction gap determines customer satisfaction; and finally, the place brand performance gap determines perceived quality, as discussed in Chapter 8 . This is supported directly by Bigné et al. (2001) and Oliver (1999) as they explain the difference between quality and satisfaction, the former being more specific, about particular attributes and key aspects of service delivery, while the latter is based on more holistic judgements based on predictions. In other words, one focuses on 'image' while the other focuses on perceived experience of actual service delivery. The impact of both of these on behavioural aspects such as repeat visits and willingness to recommend the place to others, are more conative elements that are outside the range of 
this book. From the perceived image perspective, the emphasis in this book lies primarily on assessing place image pre-visit and on evaluating alternative methods for doing so, both of which are major gaps in existing research. Sources of pre-visit image (induced, organic or autonomous agents) and differences according to cultural, social and personal characteristics will be studied in the next few chapters. 